\title{
Improving kinematic and structural performance of Geneva mechanisms using the optimal control method
}

\author{
J-J Lee* and C-C Cho
}

Department of Mechanical Engineering, National Taiwan University, Taipei, Taiwan

\begin{abstract}
A method is proposed to improve the performance of the conventional Geneva mechanism. Rather than driving the input crank at a uniform speed, this method uses optimal control theory to synthesize the speed of the crank. In this paper, design criteria are first developed based upon the elimination of the impact loading at the beginning and end of the motion cycle. In addition, three approaches to program the crank speed are introduced. In the first approach, the crank speed is designed to be optimal so as to minimize the output acceleration. In the second approach, the crank speed is determined by minimizing the input motor torque. The third approach uses the degree of wear between the crank pin and wheel slot as the index to optimize the crank speed. All design objectives are formulated as a parameterized optimization problem and solved via an efficient numerical method. Furthermore, trade-offs among the desired characteristics are taken into account by formulating the problem as a multiobjective optimization problem. Two examples are given to illustrate the design procedure.
\end{abstract}

Keywords: Geneva mechanism, optimal control, parameterized optimization, multiobjective optimization

\section{NOTATION}

$c_{1}, c_{2}, c_{3}$

$C$

$f_{\mathrm{c}}$

$f^{\prime}, f^{\prime \prime}$

$F_{\mathrm{c}}$

$I_{1}, I_{2}$

$J_{1}, J_{2}, J_{3}$

$L$

$m$

$n$

$P_{1}$

$r$

$R$

$t$ weighting factors

upper value for input jerk

force of the pin perpendicular to the slot

$\mathrm{d} f / \mathrm{d} \theta_{\mathrm{d}}, \mathrm{d} f^{\prime} / \mathrm{d} \theta_{\mathrm{d}}$

normalized pin force perpendicular to the

slot

moment of inertia of the input and the

output

objective functions

Lagrangian

degree of interpolation polynomials

number of slots

prescribed curve to be followed by the

output wheel and the input crank

length of the input crank

centre distance between the crankshaft

axis and the wheel axis

time

$t_{\mathrm{m}}$
$T$
$v$
$v_{\mathrm{d}}, v_{\mathrm{w}}$
$V$
$w_{i}$
$\beta_{0}$
$\Gamma_{\mathrm{d}}, \dot{\theta}_{\mathrm{d}}, \ddot{\theta}_{\mathrm{d}}$
$\theta_{\mathrm{w}}, \dot{\theta}_{\mathrm{w}}, \ddot{\theta}_{\mathrm{w}}$
$\Theta_{\mathrm{d}}, \dot{\Theta}_{\mathrm{d}}, \ddot{\Theta}_{\mathrm{d}}$
$\Theta_{\mathrm{w}}, \dot{\Theta}_{\mathrm{w}}$,
$\ddot{\Theta}_{\mathrm{w}}$
$\rho$

$\tau_{\mathrm{d}}$

$\tau_{\mathrm{W}}$

$\phi_{0}$ total time interval for the crank motion normalized time

sliding velocity of the pin in the slot

velocities of coincident point $\mathrm{Q}$ belonging to the crank and the wheel normalized sliding velocity of crank pin weighting factors used in multi-objective function

angle measured from the centre-line to the initial wheel position

normalized driving torque

angular displacement, velocity and

acceleration of the crank

angular displacement, velocity and

acceleration of the wheel

normalized angular displacement, velocity and acceleration of the crank

normalized angular displacement, velocity and acceleration of the wheel

centre distance of the wheel axis to the pin centre Q

driving torque

torque exerted on the wheel

angle measured from the centre-line to the initial crank position
* Corresponding author: Department of Mechanical Engineering,

National Taiwan University, 1 Roosevelt Road, Section 4, Taipei, Taiwan 106. 


\section{INTRODUCTION}

Geneva mechanisms have been widely used in the automation area where intermittent motion is required. The conventional mechanism contains a driving crank and a slotted wheel. The crank rotates at a constant speed and the wheel outputs an intermittent rotary motion. Thus, the main advantage of the Geneva mechanism lies in its simple structure. However, its main disadvantage is the non-zero acceleration at positions where the roller on the crank initially engages and finally disengages with the slot in the wheel. This non-zero acceleration at initial and final positions causes an impact loading on the mechanism and therefore limits the mechanism to low-speed applications or places where smooth and quiet operations are not required. In order to eliminate the impact loading caused by nonzero initial and final accelerations, several methods have been studied. Dijksman [1] and Bagci [2] combined linkage with the Geneva wheel as a compound mechanism. Dijksman [3] and Yang and Hsia [4] used gears to form the geared Geneva wheel. Fenton et al. [5] introduced the Geneva mechanism connected in series. Besides the use of compound mechanisms, some researchers worked on changing the shape of the slot in the wheel $[6,7]$ or the shape of the crank pin $[\mathbf{8}]$. Cheng and Lin [9] proposed installing a non-linear spring element in the crank pin to reduce the shock of the wheel. All of these methods focused on redesigning the geometry of the mechanism. However, instead of altering the structure of the Geneva mechanism, the current method presents an approach to finding an appropriate input speed trajectory that not only eliminates the impact loading but also reveals good kinematic and structural properties. The method employed for the development of such an integrated approach is optimal control theory. To begin, equations of motion will be developed for the Geneva mechanism in terms of normalized parameters. The kinematic constraints for impact-free motion are then established.
Subsequently, the cost functions for kinematic and structural performance are developed followed by the solution of the problem. Finally, design examples are given to illustrate the procedure and discussions of the results are presented.

\section{MOTION AND CONSTRAINT EQUATIONS}

Figure 1 shows the schematic diagram of a traditional Geneva mechanism where $\mathrm{O}_{1}$ and $\mathrm{O}_{2}$ are respectively the centres of rotation of the crank and wheel. In the figure, $R$ is the centre distance between two rotating axes of crank $\mathrm{O}_{1} \mathrm{G}$ and wheel $\mathrm{O}_{2} \mathrm{G}$ and $r$ is the length of the input crank. The normalized displacement equation of the wheel, $\Theta_{\mathrm{w}}$, can be expressed in terms of the normalized crank input, $\Theta_{\mathrm{d}}$, as (see Appendix 1 for the derivation)

$$
\Theta_{\mathrm{w}}=F\left(\Theta_{\mathrm{d}}\right)
$$

By taking the time derivative of equation (1) once and twice, the normalized velocity and acceleration equations of the wheel respectively are obtained as

$$
\begin{aligned}
& \dot{\Theta}_{\mathrm{w}}=F^{\prime}\left(\Theta_{\mathrm{d}}\right) \dot{\Theta}_{\mathrm{d}} \\
& \ddot{\Theta}_{\mathrm{w}}=F^{\prime \prime}\left(\Theta_{\mathrm{d}}\right) \dot{\Theta}_{\mathrm{d}}^{2}+F^{\prime}\left(\Theta_{\mathrm{d}}\right) \ddot{\Theta}_{\mathrm{d}}
\end{aligned}
$$

where $\quad F^{\prime}\left(\Theta_{\mathrm{d}}\right)=\mathrm{d} F / \mathrm{d} \Theta_{\mathrm{d}} \quad$ and $\quad F^{\prime \prime}\left(\Theta_{\mathrm{d}}\right)=\mathrm{d} F^{\prime} / \mathrm{d} \Theta_{\mathrm{d}}$. Obviously, if the crank is driven at a constant speed, then the output velocity and acceleration become

$$
\begin{aligned}
& \dot{\Theta}_{\mathrm{w}}(T)=F^{\prime}\left(\Theta_{\mathrm{d}}\right) \\
& \ddot{\Theta}_{\mathrm{w}}(T)=F^{\prime \prime}\left(\Theta_{\mathrm{d}}\right)
\end{aligned}
$$

Plots of equations (3a) and (3b) are shown respectively in Figs $2 \mathrm{a}$ and $\mathrm{b}$. It can be seen that non-zero $F^{\prime \prime}\left(\Theta_{\mathrm{d}}\right)$ at the initial and final positions results in nonzero accelerations of the wheel at the corresponding positions. In what follows, a method will be proposed for synthesizing the motion of the crank that eliminates

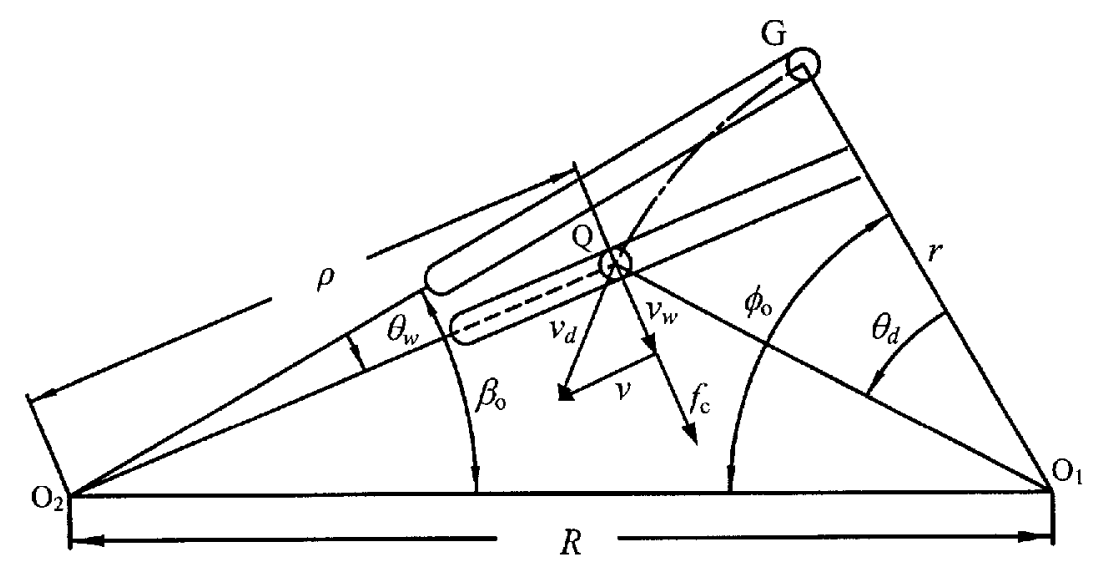

Fig. 1 Basic element of the Geneva mechanism 


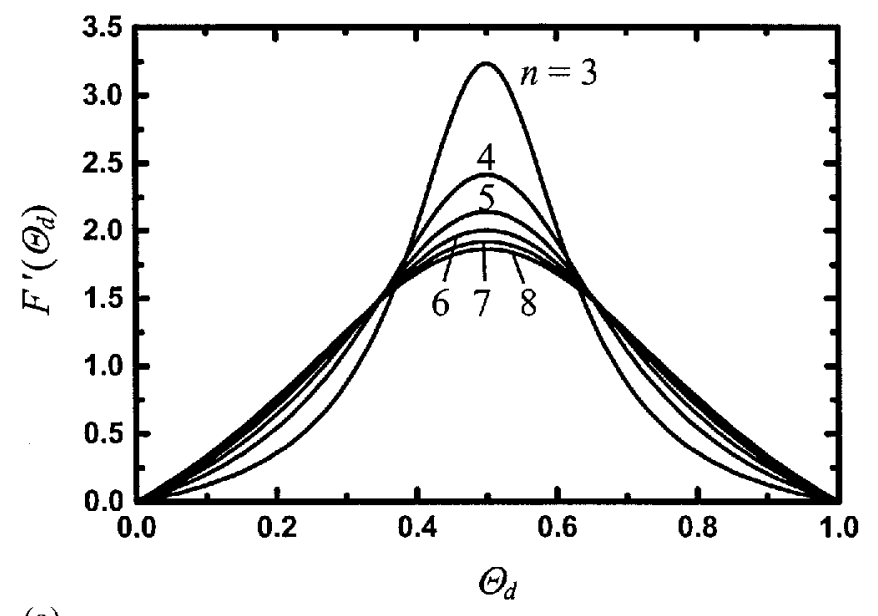

(a)

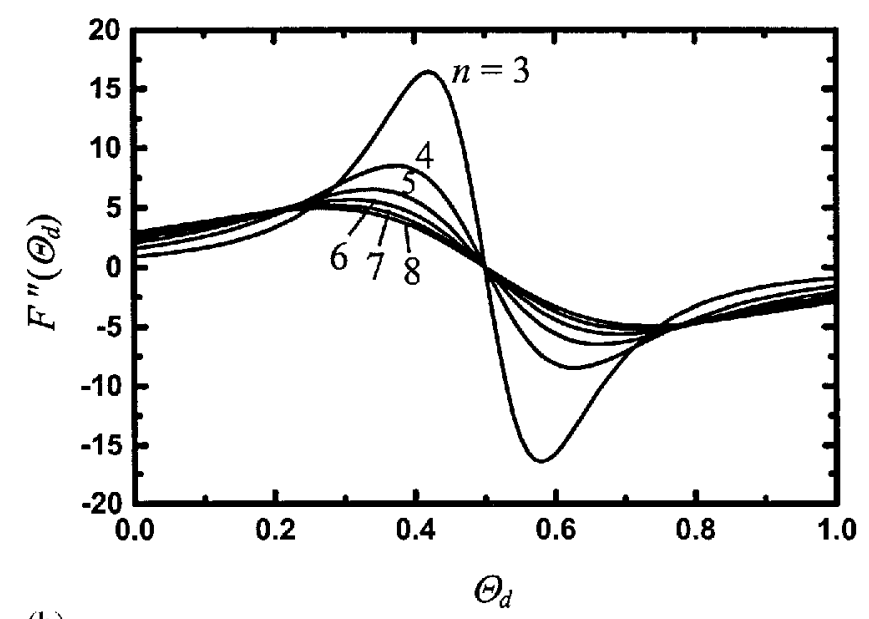

(b)

Fig. 2 (a) Plot of $F^{\prime}\left(\Theta_{\mathrm{d}}\right), n=$ number of slots, (b) Plot of $F^{\prime \prime}\left(\Theta_{\mathrm{d}}\right), n=$ number of slots

the discontinuity of the wheel acceleration. In addition, the mechanism must exhibit good kinematic and structural properties, such as low wheel acceleration, low input motor torque and/or low wear between the crank pin and the wheel slot.

1. It is first noticed that the displacement of $\Theta_{\mathrm{d}}(T)$ must satisfy the boundary conditions at the initial and final positions, i.e.

$$
\begin{aligned}
& \Theta_{\mathrm{d}}(0)=0 \\
& \Theta_{\mathrm{d}}(1)=1
\end{aligned}
$$

2. It is practical to consider that the input crank does not reverse the direction of rotation during operation, i.e.

$$
\dot{\Theta}_{\mathrm{d}}(T) \geqslant 0, \quad 0 \leqslant T \leqslant 1
$$

3. To ensure that the undesirable impact loading is completely eliminated, the accelerations of the wheel at the beginning and ending of each motion cycle must be zero. The following conditions, therefore, must be satisfied:

$$
\ddot{\Theta}_{\mathrm{w}}(0)=\ddot{\Theta}_{\mathrm{w}}(1)=0
$$

Considering this equation and noting that $F^{\prime}\left(\Theta_{\mathrm{d}}\right)=$ 0 and $F^{\prime \prime}\left(\Theta_{\mathrm{d}}\right) \neq 0$ at both positions $\Theta_{\mathrm{d}}=0$ and 1 , the following constraint equations are obtained:

$$
\begin{aligned}
\dot{\Theta}_{\mathrm{d}}(0) & =0 \\
\dot{\Theta}_{\mathrm{d}}(1) & =0
\end{aligned}
$$

4. With regards to the continuity of $\ddot{\Theta}_{\mathrm{d}}(T)$, from equations (6) and (5) the slope of $\dot{\Theta}_{\mathrm{d}}(T)$ will necessarily be zero at $T=0$ and $T=1$, i.e.

$$
\begin{aligned}
& \ddot{\Theta}_{\mathrm{d}}(0)=0 \\
& \ddot{\Theta}_{\mathrm{d}}(1)=0
\end{aligned}
$$

The kinematic constraints imposed by equations (4), (5), (6) and (7) are the necessary conditions for eliminating 
the impact loading of the Geneva wheel. Any crank motion fulfilling the above constraints will be applicable. However, the goal here is to synthesize crank motions that not only extinguish impact loading but also possess good kinematic and structural properties. Therefore, a cost function that results in crank motions with desirable properties may be defined.

\section{COST FUNCTIONS IN SYNTHESIZING THE CRANK MOTIONS}

There may be many kinds of cost functions to be studied. In this work, three cost functions from both kinematic and structural viewpoints are defined:

1. The crank motion can be considered to possess favourable accelerations of both the crank and wheel. Such characteristics can avoid excessive vibration at the output as well as not deteriorating the performance of the input motor. It is reasonable, therefore, to bring about a compromise between the acceleration desired at the input crank and the output wheel. Meanwhile, a prescribed function may additionally be designated as a reference for the input and output accelerations to trace. Thus, the cost function $J_{1}$ may be defined as

$$
\begin{aligned}
\text { Minimize } J_{1}= & c_{1} \int_{0}^{1}\left[\ddot{\Theta}_{\mathrm{w}}(T)-P_{1}(T)\right]^{2} \mathrm{~d} T \\
& +c_{2} \int_{0}^{1}\left[\ddot{\Theta}_{\mathrm{d}}(T)-c_{3} P_{1}(T)\right]^{2} \mathrm{~d} T
\end{aligned}
$$

where $c_{1}, c_{2}$ and $c_{3}$ are weighting factors and $P_{1}(T)$ is the prescribed curve to be followed by the output wheel and input crank such that equations (4) to (7) are satisfied. Here, two weighting factors are used to control the crank acceleration, so that there is more flexibility in controlling the input. Generally, the function $P_{1}$ can be chosen from the cam displacement curves with good kinematic performance, e.g. the fifth-degree polynomials or the modified sinusoidal curve.

2. A second cost function is defined as minimizing the driving torque as

$$
\text { Minimize } J_{2}=\int_{0}^{1} \Gamma_{\mathrm{d}}^{2} \mathrm{~d} T
$$

where $\Gamma_{\mathrm{d}}$ is the normalized driving torque with the same boundary conditions as above (see Appendix 2 for details of the expression and derivation). This cost function considers the performance of the driving motor when the system requirements are to be fulfilled.
3. The third cost function may be defined to consider the degree of wear between the crank pin and the wheel slot. It is assumed that Coulomb friction exits between the two elements and each slot is of an equal lubrication condition. This degree of wear is proportional to the product of the pin force perpendicular to the slot and the sliding velocity of the pin in the slot. Thus, the cost function can be written as

$$
\text { Minimize } J_{3}=\int_{0}^{1}\left(F_{\mathrm{c}} V\right)^{2} \mathrm{~d} T
$$

where $F_{\mathrm{c}}$ is the normalized normal pin force and $V$ the normalized sliding velocity with the same boundary conditions as equations (4) to (7) (see Appendix 3 for the derivation).

To summarize, programming the crank motion of the Geneva mechanism becomes the optimal control problem as

$$
\text { Minimize } J_{i} \quad(i=1,2,3)
$$

subject to equations (4), (5), (6) and (7), where $J_{i}$ is the function described by equations (8), (9) or (10).

Having derived the cost functions of controlling the properties at both input and output ends, an appropriate approach of the solution now needs to be found. In general, the approaches to the numerical solutions of the optimal control problem can be divided into two major categories: one is the two-point boundary-value problem and the other is the parameter optimization problem that transcribes the system to a finite dimensional non-linear programming problem. In the former category, the approach is generally able to handle nonlinearities of the problem. However, for many practical cases, these boundary-value problems turn out to be quite complicated to solve because of difficulty in treating boundary values, especially for those systems with inequality constraints. Examples of this approach are illustrated in references [10] to [13]. In the latter category, using the parameter optimization method avoids the requirement of solving a two-point boundary-value problem. Moreover, the problem can be solved via well-developed non-linear programming algorithms. Examples of this approach are shown in references [14] and [15] where inequality constraints are involved in the system.

In view of the cost functions shown by equations (8) to (10) and the system constraints of equations (4) to (7), the equations are so highly non-linear that an efficient parameter optimization technique will be needed in the present work. Here, the pseudospectral Chebyshev method (PCM) will be applied to solve the constrained non-linear dynamic system. This method is in some way similar to the generalized Lagrange multiplier (GLM) method used by Chew and Chuang [16]. While the GLM method uses a polynomial function to approximate the 
control variable, the PCM represents the state and control variables in the optimal control problem by a set of orthogonal polynomials and further discretizing the cost function using a cell-averaging technique. The optimal control problem is therefore transformed into an algebraic non-linear programming problem and becomes easier to handle. A description of this technique can be found in Elnagar and Kazemi [17]. The details of the formulation of the present problem are given in Appendix 4. The following is a summary of the procedure:

Step 1. Approximating the state and control variables by a set of $m$ th degree interpolation polynomials as shown by equations (32a) and (32b) in Appendix 4.

Step 2. Deriving the relationship between the state vectors $\dot{x}$ and $\boldsymbol{x}$ at the Chebyshev nodes. The result transforms the system dynamics [equation (30)] into a matrix multiplication form as shown by equation (34) in Appendix 4.

Step 3. Discretizing the performance index $J$ using the cell-averaging integration rule. The approximation technique also transforms the integration form of the performance index $J$ into a matrix multiplication form as shown by equation (36) in Appendix 4.

Step 4. Solving the non-linear programming problem formed by equations (37) to (39) in Appendix 4. A commercialized package tool such as the MATLAB optimization toolbox can be used as the solution solver.

\section{RESULTS AND DISCUSSIONS}

In this section, first the results of input and output responses are investigated using the formulation described in equation (11). The results serve as a preliminary understanding of the optimal problem.

In objective $J_{1}$, function Gutman F-3 [18] is chosen for $P_{1}(T)$ and the weighting factors are given as $c_{1}=25$, $c_{2}=1, c_{3}=3$. The weighting factors are chosen such that the output acceleration has the weighting on the cost function. In objective $J_{2}$, the ratio $I_{2} / I_{1}=10$. The order of the polynomials used in the pseudospectral Chebyshev method is $m=15$ for the three design objectives. The order of the polynomials may affect the accuracy of the result. Here, the accuracy of the approximation will not be considered due to the value of the order. Influence of the order on the cost function can be referred to [17]. The kinematic and structural properties of these designs are now evaluated.

Figures $3 \mathrm{a}$ and $\mathrm{b}$ show the normalized accelerations of the crank $\left(\ddot{\Theta}_{\mathrm{d}}\right)$ and wheel $\left(\ddot{\Theta}_{\mathrm{w}}\right)$ respectively. As can be seen from Fig. 3a, the acceleration profiles for $J_{1}$ and $J_{2}$ are similar while the acceleration profile for $J_{3}$ is rapidly ascending/descending at the beginning/ending positions.
To gain some insight into the behaviour of input acceleration, the jerk profile of the input crank is observed. Figure $3 \mathrm{c}$ shows the jerk of the input crank for the three design objectives. The peak jerks for three designs are all shown to be excessively high (highest for $J_{3}$ ) at both ends of the operation. This implies that the rate of change of the input acceleration may be too large to be followed in the practical condition. Thus, from the preliminary study it may be advisable to add an inequality constraint to restrain the value of the rate of change of the input acceleration. Here, let

$$
\dddot{\Theta}_{\mathrm{d}} \leqslant C
$$

where $C$ is the upper limit for the value of the input jerk. By setting the limit of the rate of change of the input acceleration as $C=200$ and increasing the order of the polynomials to $m=38$, the optimal control system is reevaluated as follows.

Figures $4 \mathrm{a}, \mathrm{b}$ and $\mathrm{c}$ respectively show the normalized input velocity, input acceleration and output acceleration for the three designs with the control of the input jerk. From Fig. 4a, the velocity profiles of the three designs are wavy compared with that of the conventional Geneva mechanism. From Fig. 4b, it can be seen that with the added input jerk constraint, drastic changes of the input accelerations at the beginning and ending positions are alleviated. These profiles become smoother than those of the previous cases. In Fig. 4c, the profile for objective $J_{1}$ illustrates that the curve $P_{1}(T)$ has weighting on the output acceleration profile. Figures $4 d$ and e show the input torque and wear condition respectively for the three objectives. As can be seen, there is antithesis in the performance of the three design objectives. While obtaining optimal input torque $\left(J_{2}\right)$, the wear condition becomes worse, and vice versa. The motor torque is also found to be sacrificed in order to reduce the output acceleration in evaluating the profiles of $J_{1}$, though the wear condition does not become as poor as the second design $J_{2}$. Thus, a tradeoff needs to be made between the kinematic and structural properties. In such a condition, a fourth design objective with prescribed weighting factors can be defined as

$$
\begin{aligned}
\operatorname{Minimize} J_{4}= & w_{1} \frac{J_{1}-J_{1}^{*}}{J_{1, \max }-J_{1}^{*}}+w_{2} \frac{J_{2}-J_{2}^{*}}{J_{2, \max }-J_{2}^{*}} \\
& +w_{3} \frac{J_{3}-J_{3}^{*}}{J_{3, \max }-J_{3}^{*}}
\end{aligned}
$$

where $w_{i}$ are the weighting factors, $J_{i}^{*}$ are the minimal values of the objective $J_{i}$ and $J_{i, \max }$ are the relative maximum values of the objective $J_{i}$. The minimum and relative maximum values of each objective $J_{i}$ can be found from a comparison of the periods while pursuing each optimal cost function. 


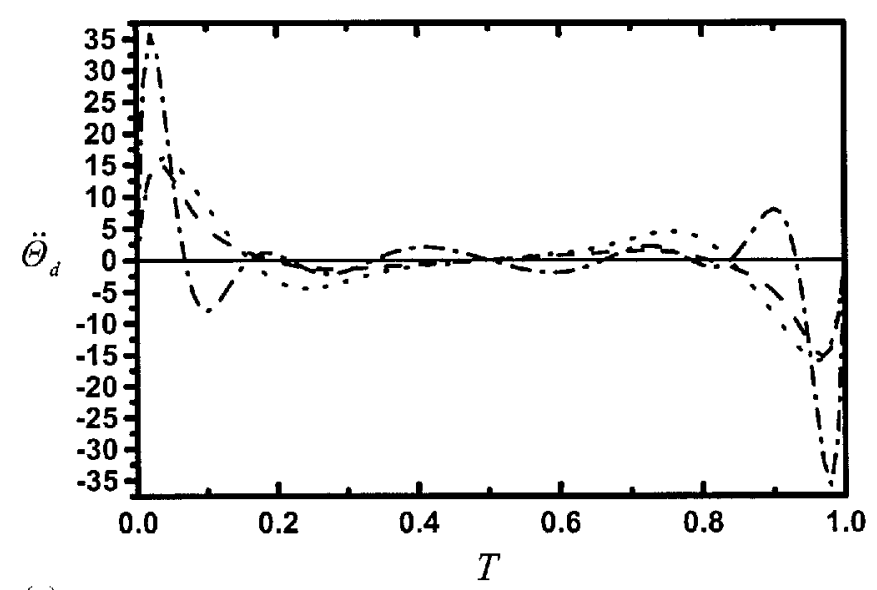

(a)

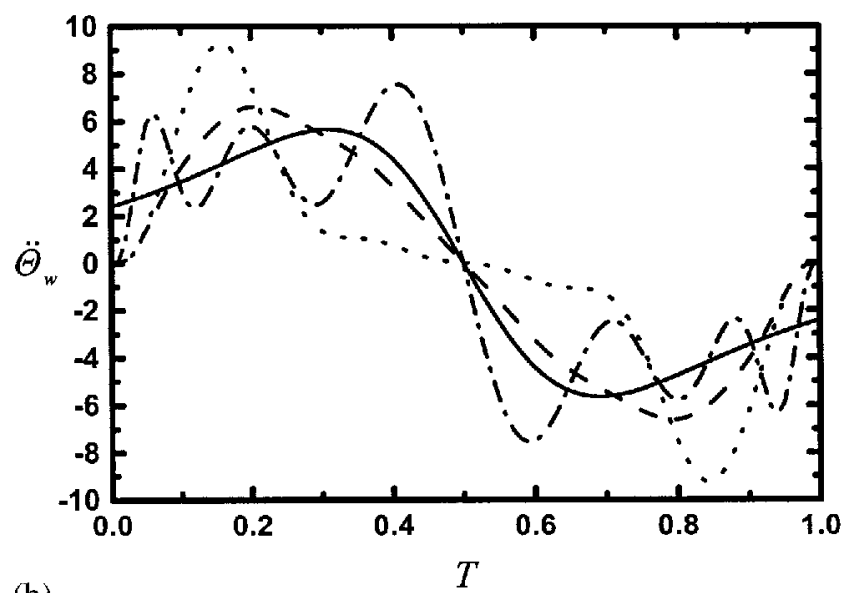

(b)

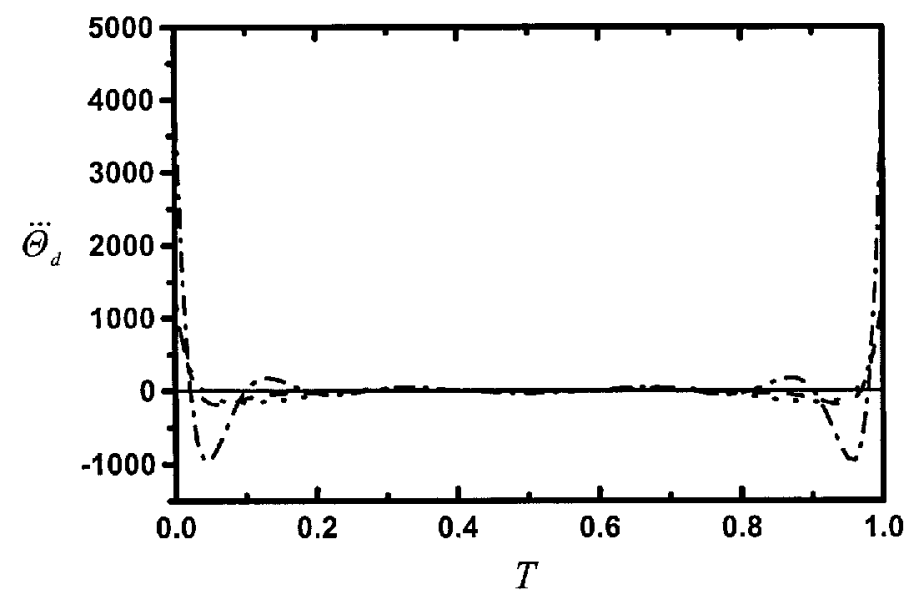

(c)

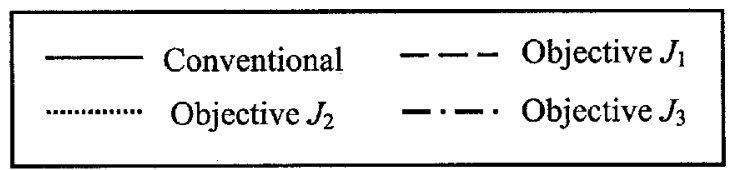

Fig. 3 (a) Input acceleration, (b) output acceleration, (c) input jerk 


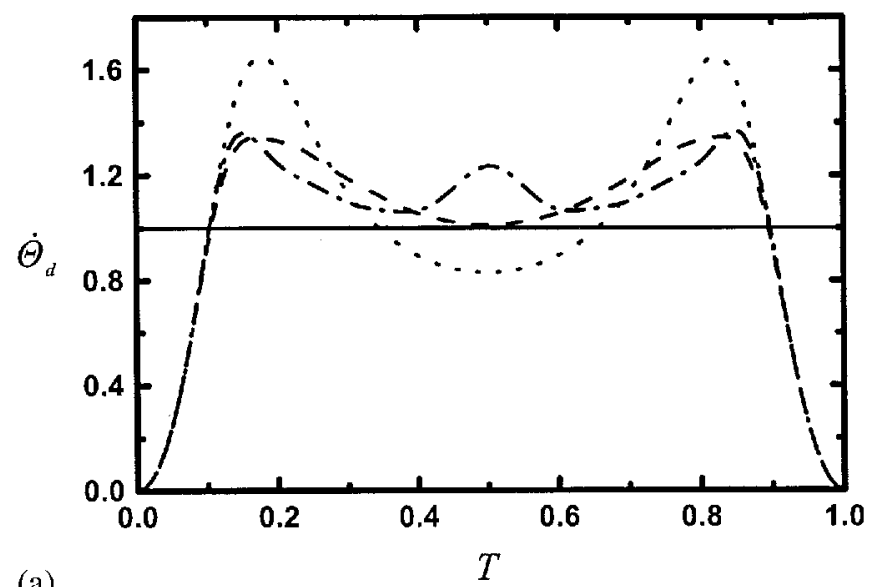

(a)

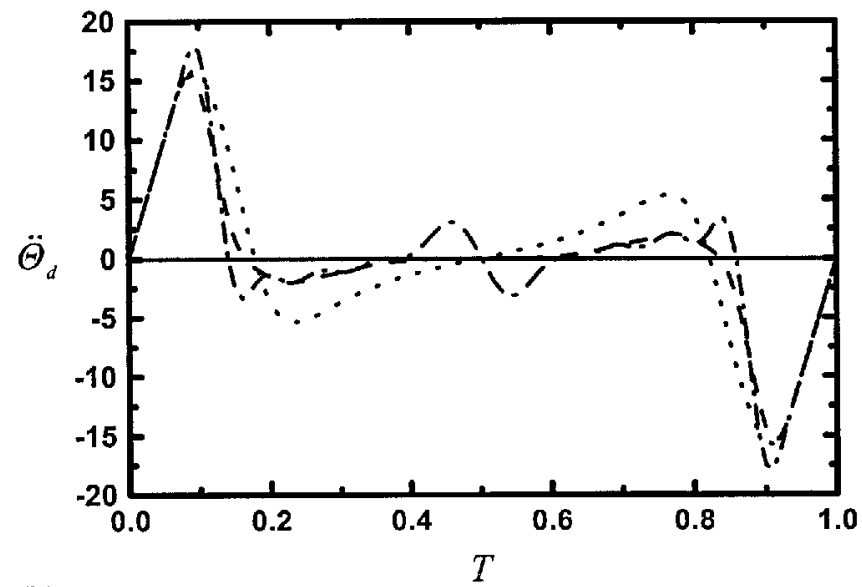

(b)

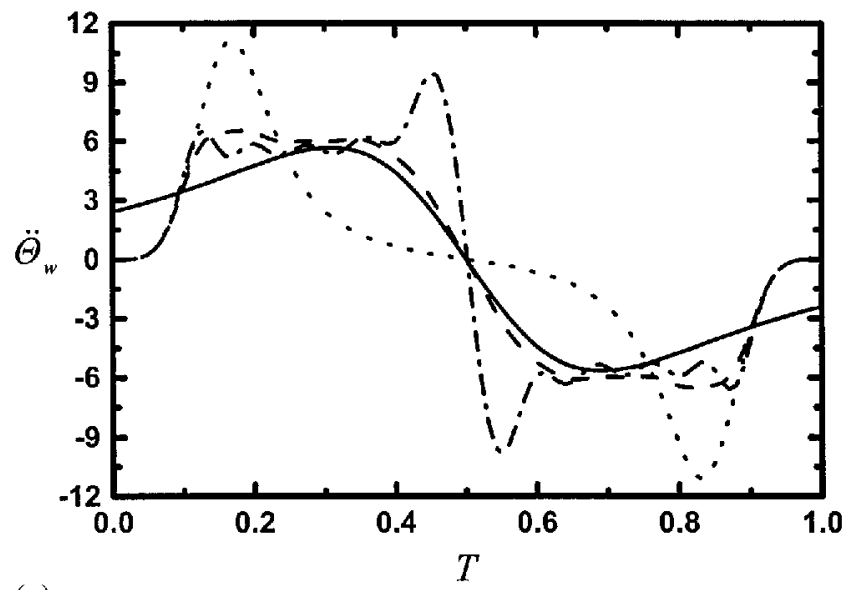

(c)

Fig. 4 (continued over) 


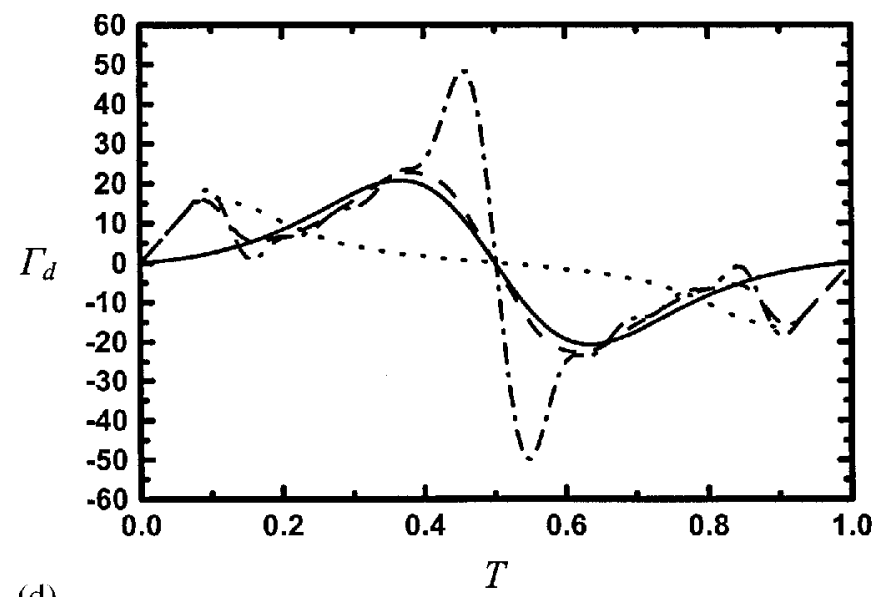

(d)

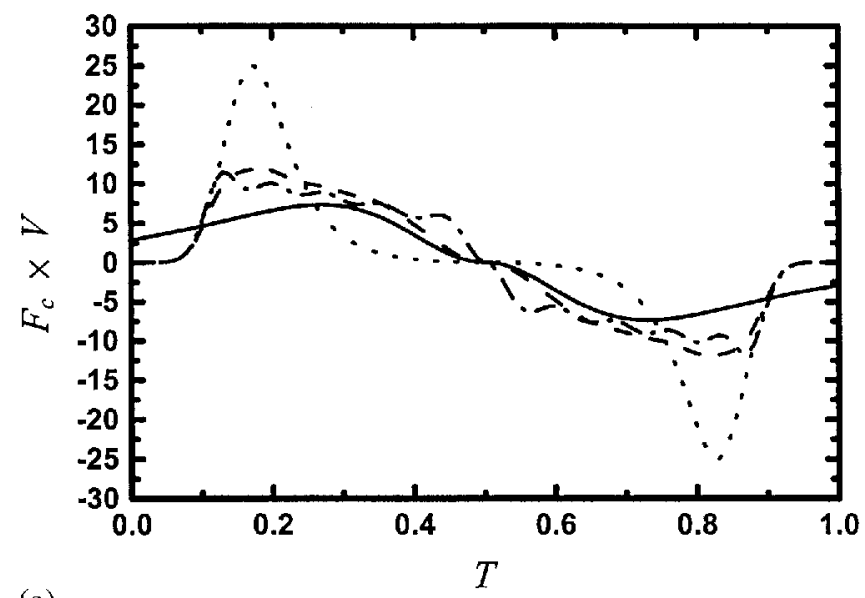

(e)

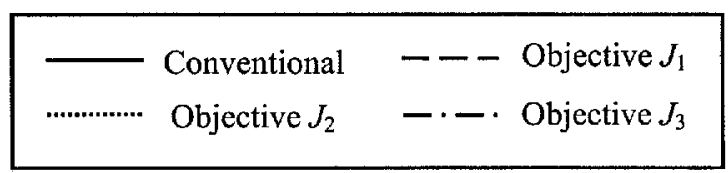

Fig. 4 (a) Input velocity, with input jerk constraint; (b) input acceleration, with input jerk constraint; (c) output acceleration, with input jerk constraint; (d) driving torque, with input jerk constraint; (e) the degree of wear $\left(F_{\mathrm{c}} V\right)$, with input jerk constraint

Table 1 lists the minimum and relative maximum values of each cost function. Thus, if a set of weighting factors is given, the compromised design objective can be obtained. Two examples have been given with weighting factors $\left(w_{1}, w_{2}, w_{3}\right)=(4,10,1)$ and $\left(w_{1}, w_{2}, w_{3}\right)=(1,3,5)$. The first case is designed to put the low input torque $\left(J_{2}\right)$ on the weighting with moderately low output acceleration $\left(J_{1}\right)$. The second case is designed to consider a larger weighting on the degree of wear $\left(J_{3}\right)$ while still keeping the other two properties not too biased. Figures $5 \mathrm{a}, \mathrm{b}, \mathrm{c}, \mathrm{d}$ and e show the input velocity, input acceleration, output acceleration, input torque and wear condition respectively for the two cases. Table 2 lists the values of cost functions for each case. It can be seen that case 1 shows a favourable output acceleration, low driving torque and a reasonable wear condition as compared with each
Table 1 The minimum and relative maximum values of each cost function

\begin{tabular}{llll}
\hline & $J_{1}$ optimized & $J_{2}$ optimized & $J_{3}$ optimized \\
\hline$J_{1}$ & $373.4085^{*}$ & $831.9899^{* *}$ & 605.8167 \\
$J_{2}$ & 387.3644 & $151.3450^{*}$ & $850.4177^{* *}$ \\
$J_{3}$ & 109.8183 & $203.3274^{* *}$ & $101.3720^{*}$ \\
\hline
\end{tabular}

*Minimum value; ** relative maximum value.

Table 2 Values of the cost functions for each of the design examples

\begin{tabular}{lll}
\hline & Optimum of case 1 & Optimum of case 2 \\
\hline$J_{1}$ & 450.6030 & 389.5073 \\
$J_{2}$ & 229.4411 & 328.5021 \\
$J_{3}$ & 126.1944 & 110.1919 \\
\hline
\end{tabular}




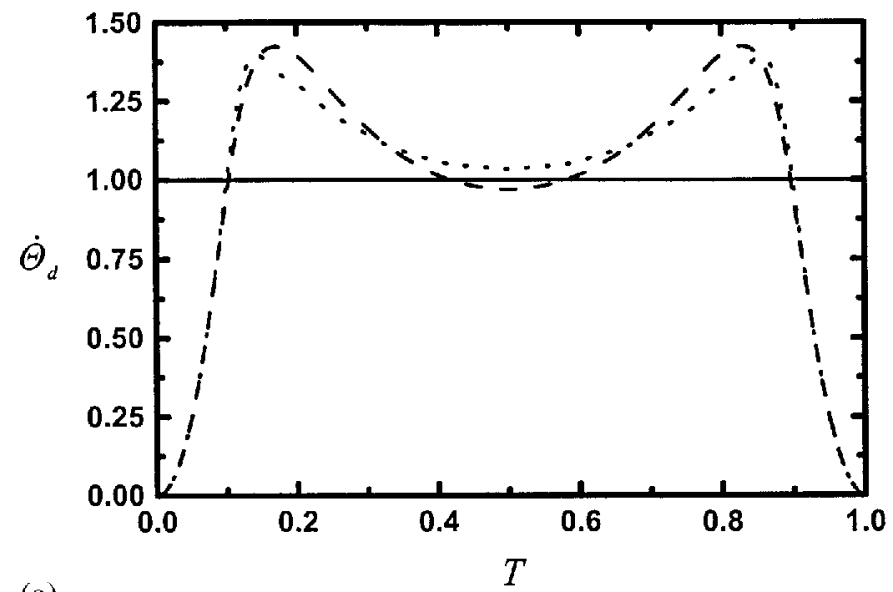

(a)

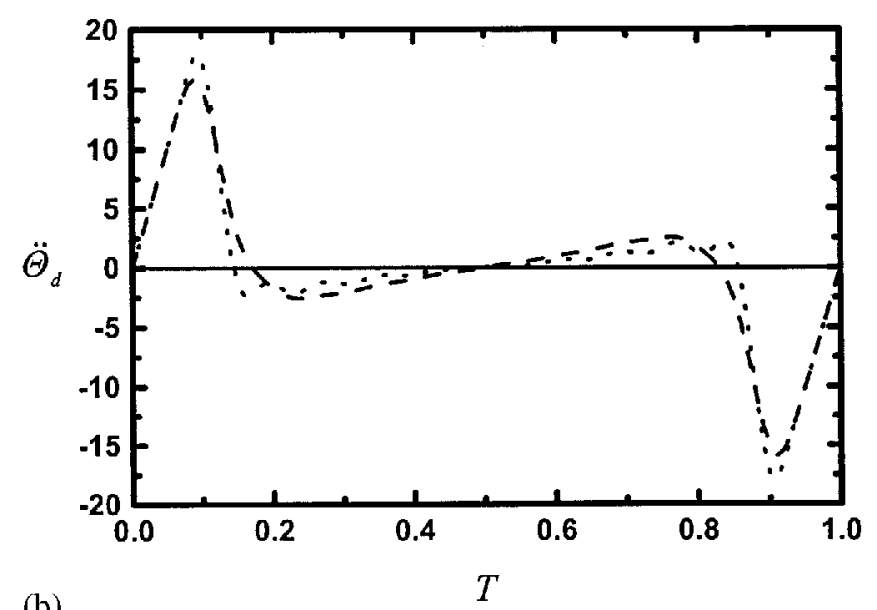

(b)

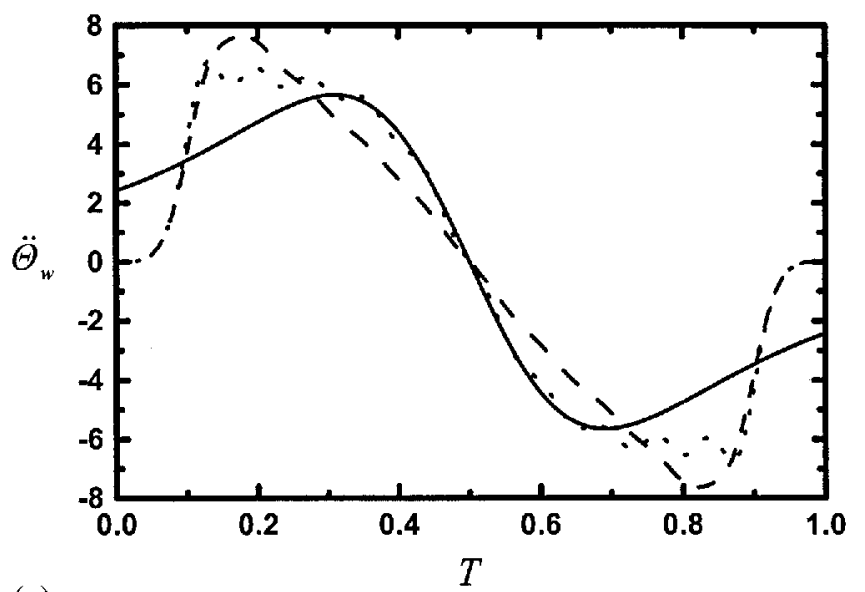

(c)

Fig. 5 (continued over) 


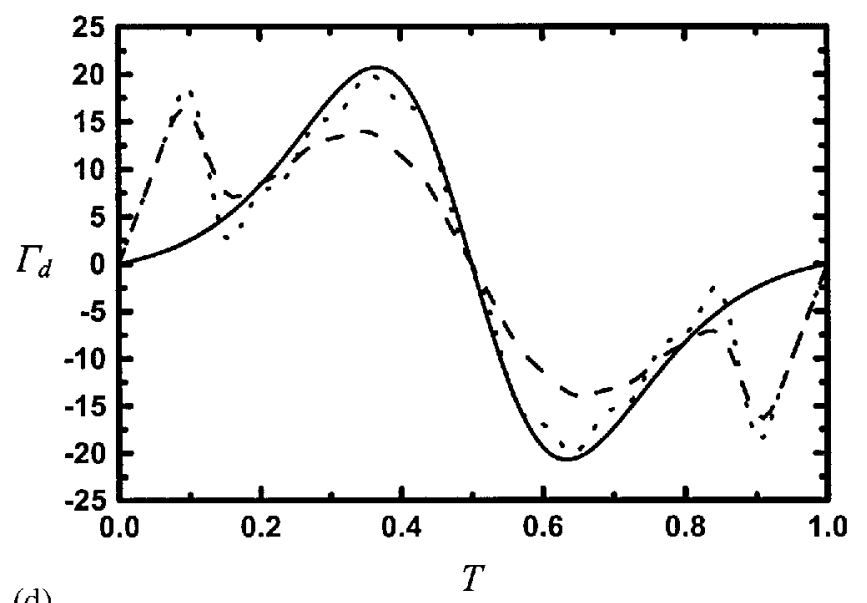

(d)

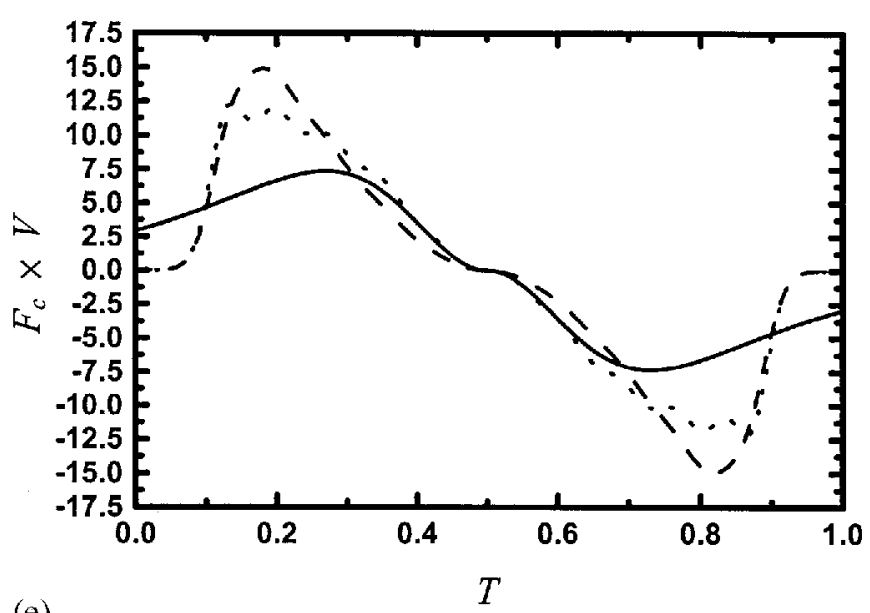

(e)

$$
\begin{aligned}
& \text { Conventional }--- \text { Objective Case } 1 \\
& \text { Objective Case } 2
\end{aligned}
$$

Fig. 5 (a) Input velocity, weighted-objective; (b) input acceleration, weighted-objective; (c) output acceleration, weighted-objective; (d) driving torque, weighted-objective; (e) the degree of wear $\left(F_{\mathrm{c}} V\right)$, weighted-objective

single objective function. As for case 2 , results show an average performance for all characteristics. Smaller peak output acceleration and a smoother wear condition are produced compared with case 1 . It can also be noted that the discontinuity of the wheel acceleration (Fig. 5c) and degree of wear (Fig. 5e) at the beginning and ending positions are diminished in both cases.

\section{CONCLUSIONS}

In this work, the feasibility of the driving Geneva mechanism via the optimal control method has been investigated. The kinematic constraints of eliminating the impact loading at the beginning and end of the motion cycle are established. In addition, cost functions for improving kinematic and structural properties are defined. The problem is formulated into an optimal control problem. The pseudospectral Chebyshev method is used to solve the optimal control problem. The results obtained have shown that by proper weight selection, the design can produce a mechanism with better kinematic and structural properties when compared with the traditional Geneva mechanism. Furthermore, the advantage of the proposed method reveals that there is no need to alter the existing structure. Future work is likely to focus on improving the dynamic performance of the mechanism, e.g. by minimizing the residual vibration. 


\section{REFERENCES}

1 Dijksman, E. A. Jerk-free Geneva wheel driving. $J$. Mechanisms, 1966, 1, 235-283.

2 Bagci, C. Synthesis of double-crank driven mechanisms with adjustable motion and dwell time ration. Mech. Mach. Theory, 1977, 12(6), 619-638.

3 Dijksman, E. A. Gear-wheel drive Geneva wheels. Rev. Roum. Sci. Techn.-Me'c. Appl., 1976, 21(4), 581-606.

4 Yang, A. T. and Hsia, L. M. Multistage geared Geneva mechanism. Trans. ASME, J. Mech. Des., 1979, 101, 4146.

5 Fenton, R. G., Zhang, Y. and Xu, J. Development of a new Geneva mechanism with improved kinematic characteristics. Trans. ASME, J. Mech. Des., 1991, 113, 40-45.

6 Al-Sabeeh, A. K. Double crank external Geneva mechanism. Trans. ASME, J. Mech. Des., 1993, 115, $666-670$.

7 Fenton, R. G. Geneva mechanisms connected in series. Trans. ASME, J. Engng for Industry, 1975, 97, 603608.

8 Sadek, K. S. H., Lloyd, J. L. and Smith, M. R. A new design of Geneva drive to reduce shock loading. Mech. Mach. Theory, 1990, 25, 589-595.

9 Cheng, C. Y. and Lin, Y. Improving dynamic performance of the Geneva mechanism using non-linear spring elements. Mech. Mach. Theory, 1995, 30, 119-129.

10 Chew, M., Freudenstein, F. and Longman, R. W. Application of optimal control theory to the synthesis of highspeed cam-follower systems. Part 1: optimality criterion. Trans. ASME, J. Mechanisms, Transmissions, and A utomn in Des., 1983, 105, 577-584.

11 Chew, M., Freudenstein, F. and Longman, R. W. Application of optimal control theory to the synthesis of highspeed cam-follower systems. Part 2: system optimization. Trans. ASME, J. Mechanisms, Transmissions, and A utomn in Des., 1983, 105, 585-591.

12 Fabien, B. C., Longman, R. W. and Freudenstein, F. The design of high-speed dwell-rise-dwell cams using linear quadratic optimal control theory. Trans. ASME, J. Mech. Des., 1994, 116, 867-874.

13 Yan, H. S. and Chen, W. R. On the output motion characteristics of variable input speed servo-controlled slider-crank mechanisms. Mech. Mach. Theory, 2000, 35, 541-561.

14 Cuthrell, J. E. and Biegler, L. T. On the optimization of differential-algebraic process systems. Am. Inst. Chem. Engrs J., 1987, 3, 1257-1270.

15 Vlassenbroeck, J. and Dooren, R. V. A Chebyshev technique for solving nonlinear optimal control problems. IEEE Trans. A utomat. Cont., 1988, 33(4), 333-340.

16 Chew, M. and Chuang, C. H. Minimizing residual vibrations in high-speed cam-follower systems over a range of speeds. Trans. ASME, J. Mech. Des., 1995, 177, 166172.

17 Elnagar, G. N. and Kazemi, M. A. Pseudospectral Chebyshev optimal control of constrained nonlinear dynamical systems. Comput. Optimization and Applics, 1998, 11, 190217.

18 Gutman, A. S. To avoid vibration-try this new cam profile. Product Engng, 1960, 32(44), 42-48.

\section{APPENDIX 1}

As Fig. 1 shows, $\beta_{0}$ is the angle between the initial position of the wheel and centre-line $\mathrm{O}_{1} \mathrm{O}_{2}$ and $\phi_{0}$ is the angle between the initial position of the crank and centre-line $\mathrm{O}_{1} \mathrm{O}_{2} ; \beta_{0}$ and $\phi_{0}$ are given as $\pi / n$ and $(\pi / 2-\pi / n)$, where $n$ is the number of slots. Let $\theta_{\mathrm{w}}$ be the angular displacement of the wheel measured from the initial position to the current position $\mathrm{O}_{2} \mathrm{Q}$ and $\theta_{\mathrm{d}}$ the angular displacement of the crank measured from the initial to the current position $\mathrm{O}_{1} \mathrm{Q}$. Then, the angular displacement $\theta_{\mathrm{w}}$ can be expressed in terms of crank input $\theta_{\mathrm{d}}$ as

$$
\theta_{\mathrm{w}}=\beta_{0}+\sin ^{-1}\left[E \sin \left(\theta_{\mathrm{d}}-\phi_{0}\right) / A\right] \equiv f\left(\theta_{\mathrm{d}}\right)
$$

where $E$ is given by $E=r / R=\sin (\pi / n)$ and $A=\left[1+E^{2}-2 E \cos \left(\theta_{\mathrm{d}}-\phi_{0}\right)\right]^{1 / 2}$.

The angular velocity of the wheel, $\dot{\theta}_{\mathrm{w}}$, can be obtained by differentiating equation (14) with respect to time:

$$
\dot{\theta}_{\mathrm{w}}=\frac{\mathrm{d} \theta_{\mathrm{w}}}{\mathrm{d} t}=\frac{\mathrm{d} f}{\mathrm{~d} \theta_{\mathrm{d}}} \frac{\mathrm{d} \theta_{\mathrm{d}}}{\mathrm{d} t}=f^{\prime}\left(\theta_{\mathrm{d}}\right) \dot{\theta}_{\mathrm{d}}
$$

where $f^{\prime}$ is defined as $\mathrm{d} f / \mathrm{d} \theta_{\mathrm{d}}$ and $\dot{\theta}_{\mathrm{d}}$ is the angular velocity of the driving crank. Similarly, the angular acceleration of the wheel, $\ddot{\theta}_{\mathrm{w}}$, can be obtained by further differentiating equation (15) with respect to time:

$$
\begin{aligned}
\ddot{\theta}_{\mathrm{w}} & =\frac{\mathrm{d} f^{\prime}\left(\theta_{\mathrm{d}}\right)}{\mathrm{d} t} \dot{\theta}_{\mathrm{d}}+f^{\prime}\left(\theta_{\mathrm{d}}\right) \frac{\mathrm{d} \dot{\theta}_{\mathrm{d}}}{\mathrm{d} t} \\
& =f^{\prime \prime}\left(\theta_{\mathrm{d}}\right) \dot{\theta}_{\mathrm{d}}^{2}+f^{\prime}\left(\theta_{\mathrm{d}}\right) \ddot{\theta}_{\mathrm{d}}
\end{aligned}
$$

where $f^{\prime \prime} \equiv \mathrm{d} f^{\prime} / \mathrm{d} \theta_{\mathrm{d}}$ and $\ddot{\theta}_{\mathrm{d}}$ is the angular acceleration of the driving crank.

\section{Normalization of motion equations}

Let $2 \beta_{0}$ be the total angular displacement of the wheel as the crank rotates through an angle $2 \phi_{0}$ in the time period $t_{\mathrm{m}}$. Then, $T=t / t_{\mathrm{m}}$ is the normalized time, $\Theta_{\mathrm{d}}=$ $\theta_{\mathrm{d}} /\left(2 \phi_{0}\right)$ the normalized angular displacement of the crank and $\Theta_{\mathrm{w}}=\theta_{\mathrm{w}} /\left(2 \beta_{0}\right)$ the normalized angular displacement of the wheel. The normalized domain and the actual domain can be summarized as

$$
\begin{array}{lll}
0 \leqslant T \leqslant 1 & \text { versus } & 0 \leqslant t \leqslant t_{\mathrm{m}} \\
0 \leqslant \Theta_{\mathrm{d}} \leqslant 1 & \text { versus } & 0 \leqslant \theta_{\mathrm{d}} \leqslant 2 \phi_{0} \\
0 \leqslant \Theta_{\mathrm{w}} \leqslant 1 & \text { versus } & 0 \leqslant \theta_{\mathrm{w}} \leqslant 2 \beta_{0}
\end{array}
$$


Parameters between the normalized motion and the actual motion of the crank can be related as

$$
\begin{aligned}
& \theta_{\mathrm{d}}=2 \phi_{0} \Theta_{\mathrm{d}} \\
& \dot{\theta}_{\mathrm{d}}(t)=\left(2 \phi_{0} / t_{\mathrm{m}}\right) \dot{\Theta}_{\mathrm{d}}(T) \\
& \ddot{\theta}_{\mathrm{d}}(t)=\left(2 \phi_{0} / t_{\mathrm{m}}^{2}\right) \ddot{\Theta}_{\mathrm{d}}(T)
\end{aligned}
$$

Similarly, the relations between the normalized motion and the actual motion of wheel are

$$
\begin{aligned}
& \theta_{\mathrm{w}}=2 \beta_{0} \Theta_{\mathrm{w}} \\
& \dot{\theta}_{\mathrm{w}}(t)=\left(2 \beta_{0} / t_{\mathrm{m}}\right) \dot{\Theta}_{\mathrm{w}}(T) \\
& \ddot{\theta}_{\mathrm{w}}(t)=\left(2 \beta_{0} / t_{\mathrm{m}}^{2}\right) \ddot{\Theta}_{\mathrm{w}}(T)
\end{aligned}
$$

\section{APPENDIX 2}

\section{Normalized driving torque $\Gamma_{\mathrm{d}}$}

Let $I_{1}$ and $I_{2}$ respectively be the input and output moments of inertia of the Geneva mechanism. Then using the Lagrange equation approach, the required driving torque, $\tau_{\mathrm{d}}$, can be formulated as

$$
\frac{\mathrm{d}}{\mathrm{d} t}\left(\frac{\partial L}{\partial \dot{\theta}_{\mathrm{d}}}\right)-\frac{\partial L}{\partial \theta_{\mathrm{d}}}=\tau_{\mathrm{d}}
$$

where the Lagragian, $L$, is given as $L=\frac{1}{2} I_{1} \dot{\theta}_{\mathrm{d}}^{2}+\frac{1}{2} I_{2} \dot{\theta}_{\mathrm{w}}^{2}$. From equation (2) and noting $\mathrm{d} \dot{\theta}_{\mathrm{w}} / \mathrm{d} \dot{\theta}_{\mathrm{d}}=f^{\prime}\left(\theta_{\mathrm{d}}\right)$ and $\mathrm{d} \dot{\theta}_{\mathrm{w}} / \mathrm{d} \theta_{\mathrm{d}}=f^{\prime \prime}\left(\theta_{\mathrm{d}}\right) \dot{\theta}_{\mathrm{d}}$, the following relations can be obtained:

$$
\begin{aligned}
& \frac{\mathrm{d}}{\mathrm{d} t}\left(\frac{\partial L}{\partial \dot{\theta}_{\mathrm{d}}}\right)=I_{1} \ddot{\theta}_{\mathrm{d}}+I_{2}\left[\ddot{\theta}_{\mathrm{w}} f^{\prime}\left(\theta_{\mathrm{d}}\right)+\dot{\theta}_{\mathrm{w}} \dot{\theta}_{\mathrm{d}} f^{\prime \prime}\left(\theta_{\mathrm{d}}\right)\right] \\
& \frac{\partial L}{\partial \theta_{\mathrm{d}}}=I_{2} \dot{\theta}_{\mathrm{w}} \dot{\theta}_{\mathrm{d}} f^{\prime \prime}\left(\theta_{\mathrm{d}}\right)
\end{aligned}
$$

Substituting equations (20a) and (20b) into equation (19) yields

$$
\tau_{\mathrm{d}}(t)=I_{1} \ddot{\theta}_{\mathrm{d}}+I_{2} f^{\prime}\left(\theta_{\mathrm{d}}\right) \ddot{\theta}_{\mathrm{w}}
$$

From equations (18c), (18f) and $F^{\prime}\left(\Theta_{\mathrm{d}}\right)=$ $\left(\phi_{0} / \beta_{0}\right) f^{\prime}\left(\theta_{\mathrm{d}}\right)$, equation (21) can be rewritten as

$$
\tau_{\mathrm{d}}(T)=\frac{2 I_{1} \phi_{0}}{t_{\mathrm{m}}^{2}}\left[\ddot{\Theta}_{\mathrm{d}}+\frac{\beta_{0}^{2}}{\phi_{0}^{2}} \frac{I_{2}}{I_{1}} F^{\prime}\left(\Theta_{\mathrm{d}}\right) \ddot{\Theta}_{\mathrm{w}}\right]
$$

Thus, the normalized driving torque, $\Gamma_{\mathrm{d}}$, can be defined as

$$
\Gamma_{\mathrm{d}}=\frac{\tau_{\mathrm{d}}}{2 I_{1} \phi_{0} / t_{\mathrm{m}}^{2}}
$$

\section{APPENDIX 3}

\section{Normalized normal pin force $F_{\mathrm{c}}$ and sliding velocity $V$}

(a) From Fig. 1, the torque, $\tau_{\mathrm{w}}$, exerted on the wheel can be derived as

$$
\tau_{\mathrm{w}}=f_{\mathrm{c}} \rho=I_{2} \ddot{\theta}_{\mathrm{w}}
$$

where $f_{\mathrm{c}}$ is the pin force perpendicular to the slot. Substituting equation (18f) and $\rho=R A$ into equation (24) yields

$$
f_{\mathrm{c}}=\frac{2 I_{2} \beta_{0}}{R t_{\mathrm{m}}^{2}} \frac{\ddot{\Theta}_{\mathrm{w}}}{A}
$$

Hence, the normalized normal pin force, $F_{\mathrm{c}}$, can be defined as

$$
F_{\mathrm{c}}=\frac{f_{\mathrm{c}}}{2 I_{2} \beta_{0} /\left(R t_{\mathrm{m}}^{2}\right)}
$$

(b) From Fig. 1, the magnitude of the sliding velocity of the pin in the slot, $v$, can be written as

$$
v^{2}=v_{\mathrm{d}}^{2}-v_{\mathrm{w}}^{2}
$$

where $v_{\mathrm{d}}$ and $v_{\mathrm{w}}$ are the velocities of coincident point $\mathrm{Q}$ belonging to the crank and the wheel respectively. They are given as $v_{\mathrm{d}}=r \dot{\theta}_{\mathrm{d}}=\left(2 \phi_{0} / t_{\mathrm{m}}\right) r \dot{\Theta}_{\mathrm{d}}$ and $v_{\mathrm{w}}=\rho \dot{\theta}_{\mathrm{w}}=\left(2 \beta_{0} / t_{\mathrm{m}}\right) \rho \dot{\Theta}_{\mathrm{w}}$. Substituting $r=E A$ and $\rho=R A$ into equation (27) and rearranging yields

$$
v=\frac{R}{t_{\mathrm{m}}}\left\{\left(2 \phi_{0} E \dot{\Theta}_{\mathrm{d}}\right)^{2}-\left[2 \beta_{0} A\left(2 \phi_{0} \Theta_{\mathrm{d}}\right) \dot{\Theta}_{\mathrm{w}}\right]^{2}\right\}^{1 / 2}
$$

Thus, the normalized sliding velocity, $V$, is defined as

$$
V=\frac{v}{R / t_{\mathrm{m}}}
$$

\section{APPENDIX 4}

\section{Procedure for solving the optimal problem using the pseudospectral Chebyshev method}

Define the state vector $\boldsymbol{x}=\left[\begin{array}{lll}x_{1} & x_{2} & x_{3}\end{array}\right]^{\mathrm{T}}=\left[\begin{array}{lll}\theta_{\mathrm{d}} & \dot{\theta}_{\mathrm{d}} & \ddot{\theta}_{\mathrm{d}}\end{array}\right]^{\mathrm{T}}$ and the system control variable $u=\ddot{\theta}_{\mathrm{d}}$. Then equation (11) can be transformed into the optimal control problem as

$$
\text { Minimize } J_{i}=\int_{0}^{1} Z_{i}[x(T)] \mathrm{d} T, \quad i=1,2,3
$$

subject to the differential equations

$$
\dot{x}_{1}=x_{2}, \quad \dot{x}_{2}=x_{3}, \quad \dot{x}_{3}=u
$$


with the boundary conditions $\boldsymbol{x}(0)=\left[\begin{array}{lll}0 & 0 & 0\end{array}\right]^{\mathrm{T}}, \boldsymbol{x}(1)=$ $\left[\begin{array}{lll}1 & 0 & 0\end{array}\right]^{\mathrm{T}}$ and inequality constraint $x_{2} \geqslant 0$.

\section{Solution technique}

In order to use Chebyshev nodes, the $T$-interval $(0,1)$ needs to be transformed into the $\eta$-interval $(-1,1)$ by the form of $T=(1+\eta) / 2$. The optimal problem is restated as

$$
\begin{gathered}
\text { Minimize } J_{i}=\int_{-1}^{1} Z_{i}[x(\eta)] \mathrm{d} \eta, \quad i=1,2,3 \\
\text { subject to } x_{1}^{\prime}=\frac{1}{2} x_{2}, \quad x_{2}^{\prime}=\frac{1}{2} x_{3}, \quad x_{3}^{\prime}=\frac{1}{2} u \\
x(-1)=\left[\begin{array}{lll}
0 & 0 & 0
\end{array}\right]^{\mathrm{T}}, \quad \boldsymbol{x}(1)=\left[\begin{array}{lll}
1 & 0 & 0
\end{array}\right]^{\mathrm{T}} \\
x_{2} \geqslant 0
\end{gathered}
$$

The $x_{i}(\eta)$ and $u(\eta)$ can be approximated by the $m$ th degree interpolation polynomials as

$$
\begin{aligned}
& x_{i}^{m}(\eta)=\sum_{k=0}^{m} b_{i k} \varphi_{k}(\eta), \quad i=1,2,3 \\
& u^{m}(\eta)=\sum_{k=0}^{m} d_{k} \varphi_{k}(\eta)
\end{aligned}
$$

where $b_{i k}$ and $d_{k}$ are coefficients to be determined, $\varphi_{k}(\eta)$ is defined as

$$
\varphi_{k}(\eta)=\frac{2}{m \bar{C}_{k}} \sum_{j=0}^{m} \frac{\bar{T}_{j}\left(\eta_{k}\right) \bar{T}_{j}(\eta)}{\bar{C}_{j}}, \quad k=0,1, \ldots, m
$$

with $\bar{C}_{0}=\bar{C}_{m}=2, \bar{C}_{k}=1$ for $1 \leqslant k \leqslant m-1$, the Chebyshev polynomial $\bar{T}_{j}(\eta)=\cos \left(j \cos ^{-1} \eta\right)$ and the Chebyshev nodes $\eta_{k}=\cos (\pi k / m)$. It can be verified that

$$
\begin{aligned}
x_{i}^{m}\left(\eta_{j}\right) & =b_{i j}, \quad i=1,2,3 ; j=0, \ldots, m \\
u^{m}\left(\eta_{j}\right) & =d_{j}, \quad j=0, \ldots, m
\end{aligned}
$$

The relation between $x^{m}(\eta)$ and $\dot{x}^{m}(\eta)$ at the Chebyshev nodes $\eta_{k}$ can be obtained by differentiating equation (32a). The result is a matrix form

$$
\begin{aligned}
\dot{x}^{m}\left(\eta_{k}\right) & =\left[D_{k 0} \cdots D_{k m}\right]\left[b_{0} \cdots b_{m}\right]^{\mathrm{T}} \\
& =\sum_{l=0}^{m} D_{k l} b_{l}, \quad k=0,1, \ldots, m ; l=0,1, \ldots, m
\end{aligned}
$$

where $\mathbf{D}=\left(D_{k l}\right)$ is an $(m+1) \times(m+1)$ matrix given as

$$
\begin{aligned}
\mathbf{D} & =\left(D_{k l}\right) \\
& = \begin{cases}\frac{C_{k}(-1)^{k+l}}{C_{l}\left(\eta_{k}-\eta_{l}\right)} & \text { if } k \neq l \\
\frac{2 m^{2}+1}{6} & \text { if } k=l=0 \\
-\frac{2 m^{2}+1}{6} & \text { if } k=l=m \\
-\frac{\eta_{l}}{2\left(1-\eta_{l}^{2}\right)} & \text { if } 1 \leqslant k=l \leqslant m-1\end{cases}
\end{aligned}
$$

The cell average of a function $\bar{F}(s)$ is defined to be

$$
\begin{aligned}
\int_{-1}^{1} \bar{F}(s) \mathrm{d} s= & \sum_{k=1}^{m} \int_{\eta_{k}}^{\eta_{k-1}} \bar{F}(s) \mathrm{d} s \\
= & {\left[\left(\eta_{0}-\eta_{1}\right) \cdots\left(\eta_{m-1}-\eta_{m}\right)\right]_{1 \times m}[\mathbf{B}] } \\
& \times\left[\begin{array}{c}
\bar{F}\left(\dot{\eta}_{0}\right) \\
\vdots \\
\bar{F}\left(\eta_{m}\right)
\end{array}\right]_{(m+1) \times 1}
\end{aligned}
$$

where $\mathbf{B}=\left(B_{j l}\right)$ is an $m \times(m+1)$ matrix whose elements are given as

$$
\begin{aligned}
B_{j l}=\bar{g}_{l}\left(\eta_{j-1 / 2}\right), \quad 1 \leqslant j \leqslant m, 0 \leqslant l \leqslant m \\
\eta_{j-1 / 2}=\cos \left[\frac{(j-1 / 2) \pi}{m}\right] \\
\bar{g}_{l}(\eta)=\frac{1}{C_{l} m}\left\{1+\sigma_{1} \bar{T}_{1}\left(\eta_{l}\right) U_{1}(\eta)\right. \\
\left.+\sum_{k=2}^{m} \frac{\bar{T}_{k}\left(\eta_{l}\right)\left[\sigma_{k} U_{k}(\eta)-\sigma_{k-2} U_{k-2}(\eta)\right]}{C_{k}}\right\}
\end{aligned}
$$

and

$$
\begin{array}{ll}
\sigma_{k}=\frac{\sin [(k+1) \pi /(2 m)]}{(k+1) \sin [\pi /(2 m)]}, & k=0,1,2, \ldots, m \\
U_{k}(\eta)=\frac{1}{k+1} \dot{\bar{T}}_{k+1}(\eta), & k=0,1,2, \ldots, m
\end{array}
$$

From the above procedure, formulation of equation (31) becomes

$$
\begin{aligned}
\operatorname{Minimize} J_{i}^{m}= & {\left[\left(\eta_{0}-\eta_{1}\right) \cdots\left(\eta_{m-1}-\eta_{m}\right)\right][\mathbf{B}] } \\
& \times\left[\begin{array}{c}
Z_{i}\left(b_{10}, b_{20}, b_{30}\right) \\
\vdots \\
Z_{i}\left(b_{1 m}, b_{2 m}, b_{3 m}\right)
\end{array}\right]
\end{aligned}
$$

Subject to system dynamic equations 


$$
\begin{aligned}
& {[\mathbf{D}]\left[b_{10} b_{11} \cdots b_{1 m}\right]^{\mathrm{T}}=(1 / 2)\left[b_{20} b_{21} \cdots b_{2 m}\right]^{\mathrm{T}}} \\
& {[\mathbf{D}]\left[b_{20} b_{21} \cdots b_{2 m}\right]^{\mathrm{T}}=(1 / 2)\left[b_{30} b_{31} \cdots b_{3 m}\right]^{\mathrm{T}}} \\
& {[\mathbf{D}]\left[b_{30} b_{31} \cdots b_{3 m}\right]^{\mathrm{T}}=(1 / 2)\left[d_{0} d_{1} \cdots d_{m}\right]^{\mathrm{T}}}
\end{aligned}
$$

and equality/inequality constraints

$$
\begin{aligned}
& {\left[\begin{array}{lll}
b_{10} & b_{20} & b_{30}
\end{array}\right]^{\mathrm{T}}=0} \\
& {\left[\begin{array}{lll}
b_{1 m} & b_{2 m} & b_{3 m}
\end{array}\right]^{\mathrm{T}}=0} \\
& {\left[\begin{array}{ll}
b_{20} & b_{21} \\
\cdots & b_{2 m}
\end{array}\right]^{\mathrm{T}} \geqslant 0}
\end{aligned}
$$

where $b_{i j}$ and $d_{j}$ values are to be determined. 